\title{
A study of ofloxacin and levofloxacin photostability in aqueous solutions
}

\author{
Anita Frąckowiak, Bartosz Kamiński, Bartosz Urbaniak, Paweł Dereziński, \\ Agnieszka Klupczyńska, Monika Darul-Duszkiewicz, Zenon J. Kokot
}

Department of Inorganic and Analytical Chemistry, Poznan University of Medical Sciences, Poland

\begin{abstract}
Introduction. The photostability is one of the most important properties of drugs. A comprehensive study of ofloxacin (OFX) and levofloxacin (LVX) photostability in aqueous solutions was performed. Ofloxacin is a chemotherapeutic agent belonging to the second generation fluoroquinolones and is a racemate of $(R)-(+)$ ofloxacin and (S)-(-)-ofloxacin (LVX).

Material and Methods. Samples of OFX and LVX were subjected to stress conditions of UV irradiation using a mercury-vapor lamp. The study involved development of enantioselective high-performance liquid chromatography (HPLC) and high-performance capillary electrophoresis (HPCE) methods for separation of OFX enantiomers and their degradation products. These methods were used to monitor the degradation process of OFX and LVX under irradiation and to determine the kinetics of degradation of these antibacterial agents. Moreover, the identification of photoproducts was also attempted. The structure of the main photoproducts was examined by mass spectrometry (MS).

Results and Conclusions. Using HPLC method it was possible to observe two products of OFX degradation and only one for LVX, while using HPCE method eight products of OFX degradation and six of LVX were observed. Some of the photoproducts retain character of optically active compounds. The trend of the photodegradation of both tested compounds was described by autocatalytic reaction proceeding according to the Prout-Tompkins model. Some of the products of the decomposition catalyze this reaction. The rate of degradation was similar for both enantiomers but t0.5 was slightly longer for LVX than OFX. Based on MS experiments the photodegradation products of the studied fluoroquinolones and possible pathways of UV induced decay were identified.
\end{abstract}

Keywords: fluoroquinolones, photodegradation, chiral separation.

\section{Introduction}

Ofloxacin (( \pm )-9-fluoro-2,3-dihydro-3-methyl-10-(4methyl-1-piperazinyl)-7-oxo-7H-pyrido [1,2,3-de][1,4] benzoxazine-6-carboxylic acid) is a second generation fluoroquinolone. This chemotherapeutic agent is often used for treatment of infections caused by Gram-negative and atypical bacteria [1-3]. Ofloxacin is a racemate of two enantiomers, (R)-(+)-ofloxacin and (S)-(-)-ofloxacin (levofloxacin). Antibacterial activity of levofloxacin is 8-128 times higher than that of R-(+)-ofloxacin and two times higher than that of racemic mixture [4]. The situation that one of the enantiomers (eutomer) is more active than the other (distomer) is relatively common in pharmacology. It is estimated that $40 \%$ of available drugs are chiral compounds and $25 \%$ are used as pure enantiomers. Administration of drugs in the form of a pure enantiomer is much more beneficial for many reasons. It is possible to use a lower dose, thus reducing the risk of side effects or overdosing. It should be noted that even if distomer is inactive it is still metabolized by the liver. Its elimination from the commercial formulation allows to relieve the organ 
[5-8]. Ofloxacin is used therapeutically in its racemate form and, since 1998, additionally as the enantiopure $S-(-)$ isomer [9].

The photostability of the drug is one of the most important properties of the substance which affects not only the stability of the original drug, but also its phototoxicity in the human body. Fluoroquinolones including ofloxacin induce photosensitivity in humans at very low incidence $[10,11]$. Photosensitivity includes phototoxicity and photoallergy. The phototoxic reaction is linked to exaggerated sunburn and the action spectrum of the toxicity is thought to be included in the wavelength range of UVA. The photoallergenicity of fluoroquinolones is mainly derived from their photohaptenic moiety, and photomodification of skin epidermal cells with fluoroquinolones is thought to be an initial step for this photoallergy [12-14].

The current research presents a comprehensive study of ofloxacin and levofloxacin photostability in aqueous solutions. The study involved development of enantioselective high-performance liquid chromatography (HPLC) and high-performance capillary electrophoresis (HPCE) methods for separation of OFX enantiomers as well as their degradation products. These methods were used to monitor the degradation process of OFX and LVX under UV irradiation and to determine the kinetics of degradation of these antibacterial agents. Moreover, the identification of photoproducts was also attempted.

\section{Experimental}

\section{Chemicals and reagents}

Ofloxacin, levofloxacin, sparfloxacin (SPX, IS), pipemidic acid (PIP, IS) and 2-hydroxypropyl- $\beta$-cyclodextrin (HP- $\beta$-CD) were obtained from Sigma Aldrich Co. (USA), L-isoleucine, $\mathrm{CuSO}_{4}, \mathrm{HCOONH}_{4}, \mathrm{HPLC}$ grade methanol and acetonitrile were obtained from Merck (Germany), $\mathrm{H}_{3} \mathrm{PO}_{4}, \mathrm{NaH}_{2} \mathrm{PO}_{4}, \mathrm{NaOH}$ were obtained from POCh S.A. (Poland). All chemicals used in this study were of analytical grade.

\section{HPLC method development}

An HPLC system (1200 Series, Agilent Technologies) containing a binary pump G1312A, autosampler HiP-ALS G1367B and diode array detector (DAD) G1315D $(294.5 \mathrm{~nm})$ was used. Separation of OFX, LVX and their photoproducts was performed using a reverse-phase column - Zorbax Eclipse XDB-C18 Analytical, 4.6x150 $\mathrm{mm}, 5$-Micron (Agilent Technologies) at $20^{\circ} \mathrm{C}$. Mobile phase was a mixture of: chiral mobile phase additives (CMPA):methanol 88:12 (v/v). CMPA consisted of 5 $\mathrm{mmol} / \mathrm{L} \mathrm{L}$-isoleucine and $4 \mathrm{mmol} / \mathrm{L}$ of copper (II) sulfate. The flow rate was maintained at $0.75 \mathrm{~mL} / \mathrm{min}$.

Calibration curves were constructed in the range of $5-200 \mu \mathrm{g} / \mathrm{mL}$ for both OFX and LVX with addition of PIP $(100 \mu \mathrm{g} / \mathrm{mL})$ as the internal standard. All calibration curves solutions and mobile phase were filtered through the $0.45 \mu \mathrm{m}$ nylon-membrane filters (Millipore).

Calibration curves of OFX and LVX were constructed for their area under the peak over IS peak area ratio $\left(A / A_{1 S}\right)$ as a function of drug concentrations. The results showed good linearity throughout the examined concentration ranges for both racemic mixture and S-(-)-enantiomer. The linear correlation equations were $y=0.1130 \pm 0.0010 x-0.0625 \pm 0.0086\left(R^{2}=0.999\right)$ for OFX and $y=0.1124 \pm 0.0002 x-0.0483 \pm 0.0090$ $\left(R^{2}=0.999\right)$ for LVX. For validation of the method, parameters such as selectivity, limit of detection (LOD), limit of quantification (LOQ), linearity, accuracy and precision (repeatability) were investigated (Table 1). The proposed RP-HPLC method showed good selectivity that was proved by the fact that the mobile phase chromatogram contained no peaks at the retention times corresponding to OFX and LVX.

\section{HPCE method development}

The enantioseparation analysis of ofloxacin and levofloxacin was conducted on G1600 HPCE system (Agilent Technologies), with DAD detection. A fused silica capillary was used with a total length of $64.5 \mathrm{~cm}$ and an effective length of $56 \mathrm{~cm}$. New capillary was con-

Table 1. The obtained values of limit of detection, limit of quantification and precision for HPLC and HPCE methods

\begin{tabular}{lcccc}
\hline & & LOD $[\mu \mathrm{g} / \mathrm{mL}]$ & LOQ $[\mu \mathrm{g} / \mathrm{mL}]$ & Precision (RSD, \%) \\
\hline \multirow{2}{*}{ ofloxacin } & HPLC & 0.01 & 0.03 & 0.03 \\
\cline { 2 - 5 } & HPCE & 0.07 & 0.20 & 0.06 \\
\hline \multirow{2}{*}{ levofloxacin } & HPLC & 0.01 & 0.02 & 0.03 \\
\cline { 2 - 5 } & HPCE & 0.07 & 0.20 & 0.01 \\
\hline
\end{tabular}

LOD - limit of detection, LOQ - limit of quantification, RSD - relative standard deviation, HPLC - high-performance liquid chromatography, HPCE - high-performance capillary electrophoresis 
ditioned by rinsing with $0.1 \mathrm{~mol} / \mathrm{L} \mathrm{NaOH}$ for $15 \mathrm{~min}$, methanol and deionized water for 10 minutes. Before each analysis the capillary was preconditioned according to the following schedule: $1.0 \mathrm{~mol} / \mathrm{L} \mathrm{NaOH}$ for 1.5 min., water for $1 \mathrm{~min}$ and finally background electrolyte (BGE) for $1.5 \mathrm{~min}$. Moreover, after each run the capillary post conditioning procedure was also involved: 0.1 $\mathrm{mol} / \mathrm{L} \mathrm{HCl}$, methanol and water for $1 \mathrm{~min}$. As a background electrolyte the phosphate buffer $(\mathrm{pH}=2.5)$ with 2-hydroxypropyl- $\beta$-cyclodextrin (HP- $\beta-\mathrm{CD})$ as a chiral separator was used. Phosphate buffer $(\mathrm{pH}=2.5)$ was prepared by mixing an appropriate aliquots of orthophosphoric acid solution $(50 \mathrm{mmol} / \mathrm{L})$ with sodium dihydrogen phosphate solution $(50 \mathrm{mmol} / \mathrm{L})$. The $\mathrm{pH}$ of the buffer was adjusted to 2.5 by addition of orthophosphoric acid (50 mmol/L) or sodium hydroxide $(0.1 \mathrm{~mol} / \mathrm{L})$ solutions. Afterwards, the amount of 2-hydroxypropyl- $\beta$-cyclodextrin was dissolved in phosphate buffer to obtain the final concentration $(H P-\beta-C D)$ of $40 \mathrm{mmol} / \mathrm{L}$. Finally the BGE solution was filtered through the $0.65 \mu \mathrm{m}$ nylon-membrane filters (Millipore) and degassed in ultrasonic bath for two minutes.

The samples of OFX and LVX were injected in hydrodynamic mode directly onto capillary under the pressure of $50 \mathrm{mbar}$ for 7 seconds. The enantioseparation was performed in positive mode, in electric field of $25 \mathrm{kV}$ for 30 minutes and detection was set at $296.4 \mathrm{~nm}$, at the maximum absorption of fluoroquinolone molecule. The HPCE studies were performed in the range of $10-150 \mu \mathrm{g} / \mathrm{mL}$ for both OFX and LVX in the presence of sparfloxacin (SPX) $(25 \mu \mathrm{g} / \mathrm{mL})$ as the internal standard. The calibration curves of OFX and LVX were plotted as peak areas ratio $\left(A / A_{1 S}\right)$ as a function of their concentration $(\mu \mathrm{g} / \mathrm{mL})$. It was stated that the HPCE enantioseparation method, used in presented studies exhibits good linearity in the analyzed range of concentrations and good coefficient of determination $\left(R^{2}\right)$. The calibration curves were described by following equations: $y=0.03425( \pm 0.00071) x+0.0702( \pm 0.0587), R^{2}=0.999$ for OFX and $y=0.03287( \pm 0.0007) x-0.0917( \pm 0.0593)$ $R^{2}=0.999$ for $L V X$. The method was validated and the parameters of the validation are presented in Table 1 .

\section{MS conditions}

Hybrid triple quadrupole/linear ion trap mass spectrometer 4000 QTRAP (Sciex) with electrospray ion source (TurbolonSpray source) was used. The MS measurements were carried out in positive ionization mode. Ion spray voltage, entrance potential, declustering potential were set at $5500 \mathrm{~V}, 10 \mathrm{~V}$ and $30 \mathrm{~V}$, respec- tively. Nitrogen was used as a curtain gas (10 psig). In MS/MS measurements nitrogen was used as a collision gas (medium).

\section{Photostability study}

Photodegradation study of OFX and LVX was performed following the recommendations of the ICH (1QB Photostability Testing of New Drug Substances and Products). The concentration of analyzed solutions of both $\mathrm{OFX}$ and LVX was $100 \mu \mathrm{g} / \mathrm{mL}$. The $\mathrm{pH}=4.5$ was obtained by dissolution of amounts of OFX and LVX in $2.5 \mathrm{~mL}$ of $0.1 \mathrm{~mol} / \mathrm{L} \mathrm{HCl}$ solution. Solutions were filtered through the $0.45 \mu \mathrm{m}$ nylon-membrane filters (Millipore) and irradiated for $210 \mathrm{~min}$ in a quartz cylindrical cuvette placed at a distance of $15.5 \mathrm{~cm}$ from a high pressure mercury lamp (HBO-50 NARVA, emission of UVB-UVA $=280-$ $400 \mathrm{~nm}$, intensity $\left.0.119-0.125 \mathrm{~mW} / \mathrm{cm}^{2}\right)$. The irradiation process was performed in triplicate.

During HPLC study of the photodegradation process of OFX and LVX a sample of $60 \mu \mathrm{L}$ was taken every $15 \mathrm{~min}$, mixed with $10 \mu \mathrm{l}$ of PIP $(714.51 \mu \mathrm{g} / \mathrm{ml})$ as an IS and introduced onto the column in triplicate. For HPCE study of the degradation of OFX and LVX aqueous solutions a sample of $60 \mu \mathrm{L}$ was taken every $15 \mathrm{~min}$, mixed with $10 \mu \mathrm{l}$ of SPX $(316.4 \mu \mathrm{g} / \mathrm{ml})$ as an IS and introduced into the HPCE system in triplicate. For MS analysis irradiated solutions $(0,60,120,180 \mathrm{~min})$ were evaporated to dryness (vacuum concentrator miVac Duo, Genevac). Dry residue was dissolved in $1 \mathrm{~mL}$ of ammonium formate solution $(0.005 \mathrm{~mol} / \mathrm{L}$ in water:methanol $50: 50 \mathrm{v} / \mathrm{v}$ ). Before MS analysis solutions were filtered through the $0.45 \mu \mathrm{m}$ nylon-membrane filters (Millipore). Solutions were introduced to MS system directly from a syringe pump.

\section{Results and Disscusion}

The enantioseparation of OFX and LVX and their photodegradation products were achieved by HPLC and HPCE methods.

A chiral ligand-exchange reversed-phase HPLC method for the determination of OFX- racemic mixture, LVX and monitoring of their photodegradation processes was developed. Before the irradiation three peaks were observed on the OFX chromatogram (1- pipemidic acid $10.68 \mathrm{~min}$; 2- (S)-ofloxacin $15.85 \mathrm{~min}$; 3- (R)ofloxacin $19.64 \mathrm{~min}$ ) and two on the LVX chromatogram (1- pipemidic acid $10.68 \mathrm{~min}$; 2- (S)-ofloxacin 15.85 min) (Figure 1). The complete decomposition of both drugs occurred after 150 min of irradiation (no peaks corresponding to OFX or LVX occurred). Peaks corre- 
sponding to the products of degradation (FP) on chromatograms were observed. The number of photoproducts was different for OFX and LVX. LVX decomposed with formation of only one FP (3.81 min, appeared after 90 min of irradiation) while two FPs formed in case of OFX (3.81 and $4.62 \mathrm{~min}$, appeared after $120 \mathrm{~min}$ of irradiation) (Figure 2). It was found that the compounds which were formed by the decomposition of OFX were the pair of enantiomers, while in the case of LVX a single peak was observed probably with the S-(-)- configuration. Moreover, it is supposed that after $90 \mathrm{~min}$ of the light exposure some part of LVX molecules changed spatial configuration and peak with $t_{r}=19.64$ min appeared on the chromatogram.

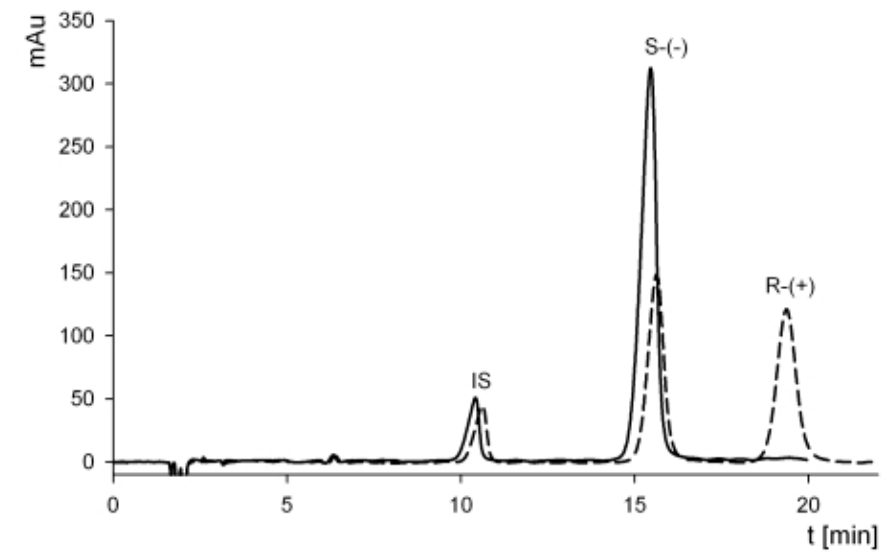

Figure 1. Chromatogram of ofloxacin (---) and levofloxacin () before the irradiation. IS internal standard, S-(-) - (S)-(-)-ofloxacin (levofloxacin), R-(+) - (R)-(+)-ofloxacin
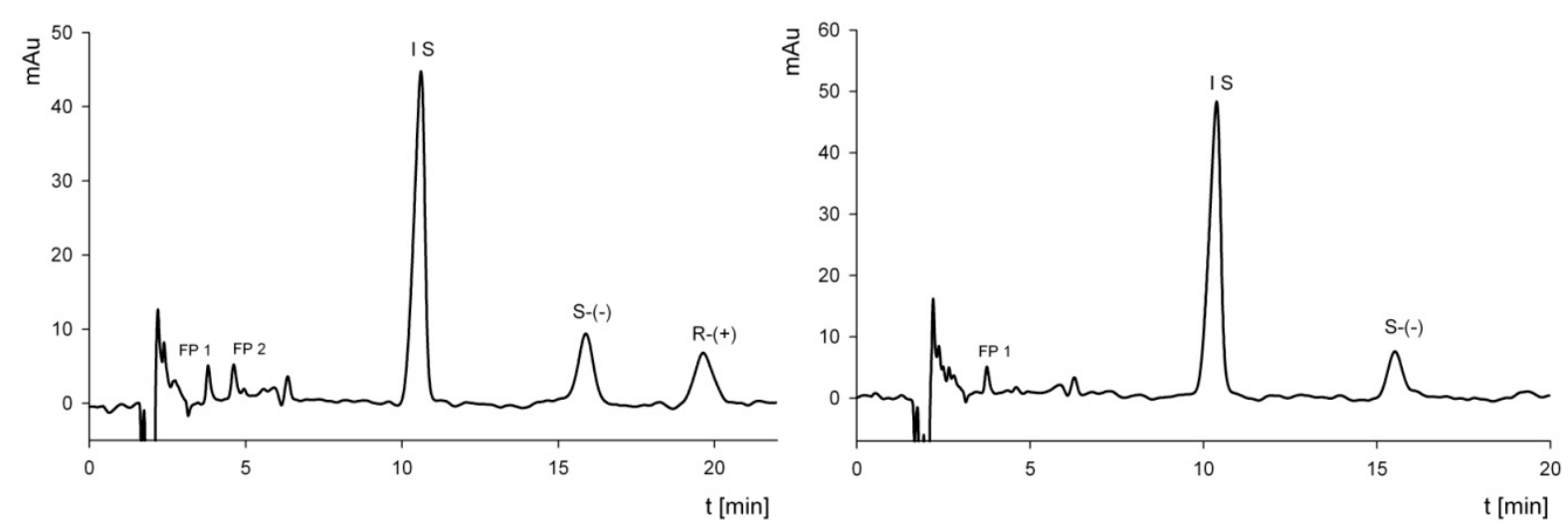

Figure 2. Chromatograms of ofloxacin (left) and levofloxacin (right) after 120 min of irradiation. FP 1 - product of degradation 1, FP 2 - product of degradation 2, IS - internal standard, S-(-) - (S)-(-)-ofloxacin (levofloxacin), R-(+) - (R)-(+)-ofloxacin

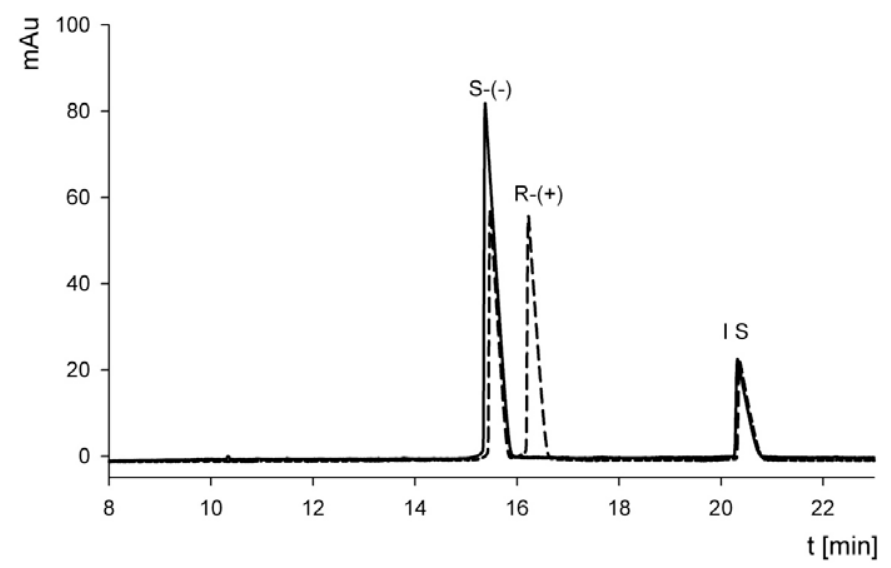

Figure 3. Electropherograms of ofloxacin (---) and levofloxacin () before the irradiation. S-(-) - (S)-(-)-ofloxacin (levofloxacin), R-(+) - (R)-(+)-ofloxacin, IS - internal standard 
In HPCE method HP- $\beta$-cyclodextrin was used as a chiral selector, and allowed to separate $S-(-)$ and $\mathrm{R}-(+)$ enantiomers from the racemic ofloxacin. Before the irradiation three peaks were observed on the OFX electropherogram (1- S-(-)-ofloxacin $15.25 \mathrm{~min}$; 2$\mathrm{R}$-(+)-ofloxacin $15.98 \mathrm{~min}$; 3- sparfloxacin as IS 20.36 min) and two on the LVX electropherogram (1- (S)ofloxacin $15.29 \mathrm{~min}$; 2- sparfloxacin as IS $20.34 \mathrm{~min}$ ) (Figure 3). It was noticed that concentration of OFX and LVX decreases over the time of irradiation, while new peaks photoproducts appeared on electropherograms. Concentration of photoproducts increased with the light exposure time (Table 2, Figure 4).

The presence of six main degradation products of LVX (A-F) and eight of OFX (B, C, C', D, D', E, E', F) was observed. Six of OFX photoproducts formed three pairs of peaks with slight differences in their migration times ( $C$ and $C^{\prime}, D$ and $D^{\prime}, E$ and $E^{\prime}$ ). On the LVX electropherogram there were observed only single peaks $(C, D$, E). In addition, in case of OFX each pair of peaks appeared after the same time of exposure. This means that the photodegradation products still retained properties of original chiral substances. It was also likely that during the photodegradation of LVX certain percentage of S-(-)-ofloxacin was converted into R-(+)-ofloxacin (FP A). It can be proved by the same migration times of FP A and R-(+)-ofloxacin.

Based on the obtained results (HPLC and HPCE) of decrease in the concentrations of examined compounds after increase of the irradiation time, the kinetics of the decomposition of OFX and LVX in aqueous solutions was determined. The rates of photodegradation reaction was time and product formation dependent - autocatalyzing reaction. The curves of concentration changes vs time were characterized by sigmoidal shape. For both of the tested compounds increasing of the reaction rate was observed in the initial stage of the reaction (15-30 $\mathrm{min}$ ) and decreasing in the final stage of exposure to light (195-210 min). The course of photodegradation of the tested compounds was best described by the second-order autocatalytic reaction equation according to the Prout-Tompkins model.

The second-order autocatalytic reaction follows the model:

$$
A \stackrel{C}{\rightarrow} C
$$

where: $A$ - substrat, $C$ - product and autocatalyst.

The concentration changes of the OFX and LVX during irradiation were described by the equation:

$$
\ln \frac{[C]}{[A]}=\ln \frac{[C]_{0}}{[A]_{0}}+\left([C]_{0}+[A]_{0}\right) k t
$$

where: $[C],[A]-$ concentration of the product and substrate respectively, $[C]_{0},[A]_{0}$, - the initial concentrations of the product and substrate respectively, $k-$ the rate constant, $t$ - time.

Table 2. Migration times [min] of ofloxacin and levofloxacin degradation products

\begin{tabular}{lcccccc}
\hline \multicolumn{1}{c}{ Degradation product } & A & B & C & D & E & F \\
\hline Irradiation time [min] & 90 & 90 & 120 & 135 & 165 & 165 \\
\hline ofloxacin & - & 14.50 & $12.87(\mathrm{C}) ; 13.02\left(\mathrm{C}^{\prime}\right)$ & $13.64(\mathrm{D}) ; 13.80\left(\mathrm{D}^{\prime}\right)$ & $13.32(\mathrm{E}) ; 13.38\left(\mathrm{E}^{\prime}\right)$ & 16.33 \\
\hline levofloxacin & 15.98 & 14.53 & 12.86 & 13.63 & 13.29 & 16.38 \\
\hline
\end{tabular}
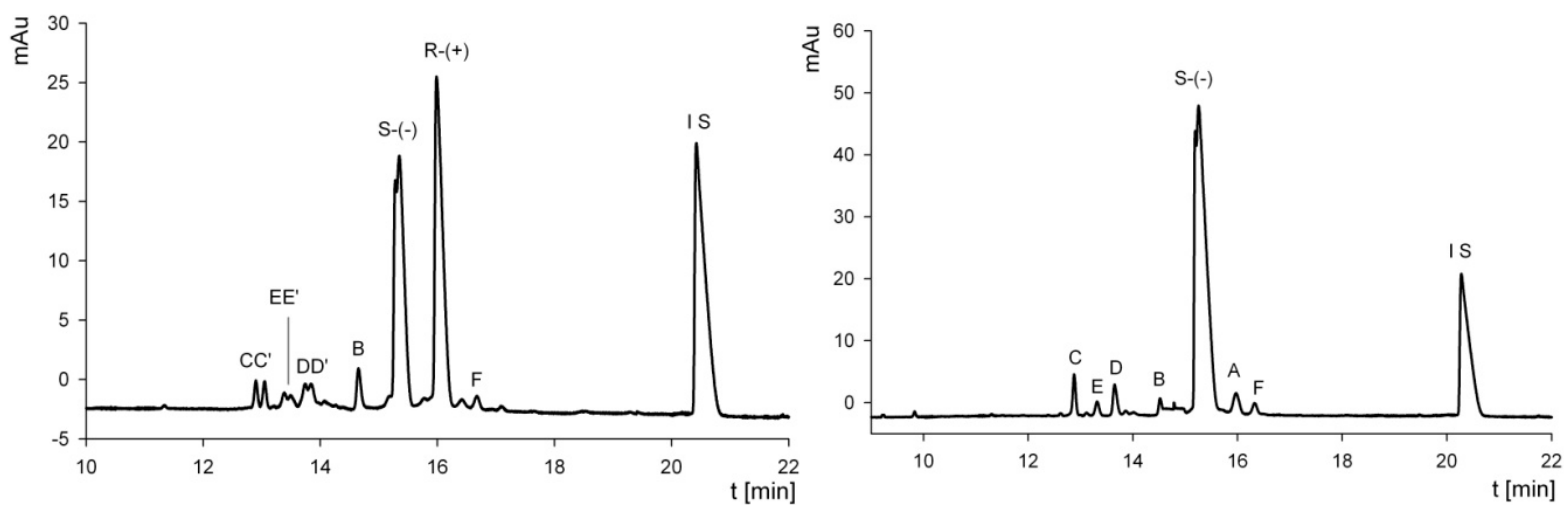

Figure 4. Electropherograms of ofloxacin (left) and levofloxacin (right) after $165 \mathrm{~min}$ of irradiation. S-(-) - (S)-(-)-ofloxacin (levofloxacin), $R-(+)-(R)-(+)$-ofloxacin, IS - internal standard, A, B, C, C', D, D', E, E', F - degradation products 
Table 3. Kinetic parameters of ofloxacin and levofloxacin photodegradation reactions determined by HPLC and HPCE methods

\begin{tabular}{|c|c|c|c|}
\hline & $\mathrm{k}\left[\mathrm{s}^{-1}\right]$ & $\mathrm{t}_{0.1}[\mathrm{~min}]$ & $\mathrm{t}_{0.5}[\mathrm{~min}]$ \\
\hline \multicolumn{4}{|c|}{ HPLC } \\
\hline ofloxacin & $5.43 \times 10^{-4}$ & 39.28 & 88.11 \\
\hline levofloxacin & $6.05 \times 10^{-4}$ & 58.86 & 105.61 \\
\hline \multicolumn{4}{|c|}{ HPCE } \\
\hline ofloxacin & $5.34 \times 10^{-6}$ & 65.28 & 130.07 \\
\hline levofloxacin & $5.76 \times 10^{-6}$ & 72.93 & 135.70 \\
\hline
\end{tabular}

HPLC - high-performance liquid chromatography, HPCE - high-performance capillary electrophoresis

Based on the theory of the second-order autocatalytic reaction the Prout-Tompkins model was used to describe the decomposition of the substance, according to the following equation:

$$
\ln \frac{c_{0}-c}{c}=\gamma t+\text { const }
$$

where: $c$ - percentage concentration of substrate, $\left(c_{0}-c\right)$ - the difference between the initial concentration of substrate and the concentration at time $t, \gamma$ - slope, const - integration constant.

Calculated kinetic parameters of OFX and LVX photodegradation processes are presented in Table 3.

The analysis of the photodegradation products of ofloxacin and levofloxacin was also performed by mass spectrometry. The spectra of fluoroquinolones before the irradiation were compared with those after 60,120 and 180 min of exposure to UV light. Peaks corresponding to the photoproducts were selected by comparing their intensity on the spectra of the solutions before and after irradiation and then an attempt was made to identify them. After 60 min of irradiation only one photoproduct of $\mathrm{m} / \mathrm{z} 336.0$ appeared. After $120 \mathrm{~min}$ of irradiation more degradation products were observed in the mass spectrum: $\mathrm{m} / \mathrm{z}$ 348.0; $\mathrm{m} / \mathrm{z} 346.0 ; \mathrm{m} / \mathrm{z} 336.0 ; \mathrm{m} / \mathrm{z} 322.0$; $\mathrm{m} / \mathrm{z} 279.0 ; \mathrm{m} / \mathrm{z} 103.0$ and $\mathrm{m} / \mathrm{z}$ 101.0. The number of the photoproducts decreased after $180 \mathrm{~min}$ of irradiation and the following peaks were detected in the spectrum: $\mathrm{m} / \mathrm{z} 346.0 ; \mathrm{m} / \mathrm{z}$ 294.0; $\mathrm{m} / \mathrm{z} 279.0$; $\mathrm{m} / \mathrm{z} 103.0$ and $\mathrm{m} / \mathrm{z} 101.0$. It should be noted that $\mathrm{m} / \mathrm{z}$ values of some FPs differed by 2 (348 and 346,103 and 101) indicating the possibility of loss of 2 hydrogens leading to the formation of double bond. Our observations were consistent with previous literature data, which pointed formation of double bonds as characteristic for UV induced degradation of fluoroquinolones [15].

In the next step, major photoproducts of the examined fluoroquinolones were fragmented using MS/MS experiments. Fragmentation made it possible to better define the structure of photoproducts. Based on the fragmentation mass spectra an attempt was made to determine the putative degradation pathways and the chemical structure of

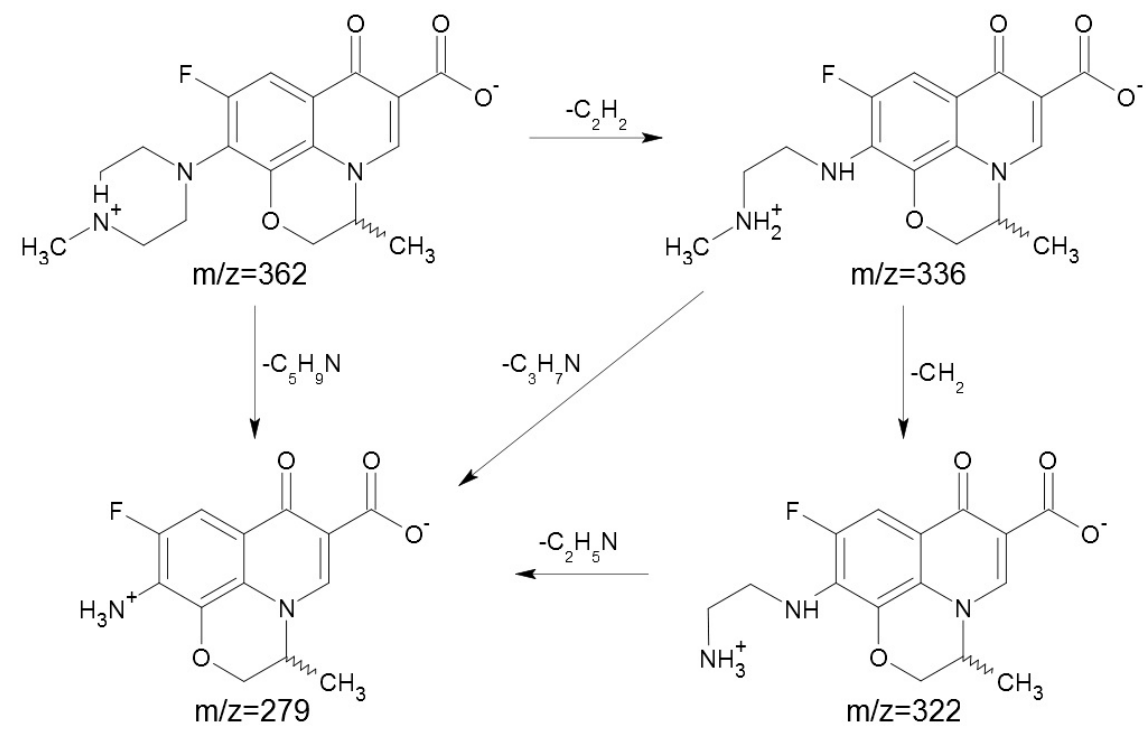

Figure 5. The diagram of ofloxacin and levofloxacin photodegradation 
the formed compounds (Figure 5). In aqueous solutions, under the influence of UV light, the piperazine ring decomposes first. On the basis of $m / z$ values of FPs it can be concluded, that disintegration proceeds by the removal of small fragments or the whole ring. In examined conditions (low pH = 4.5) there is neither fluorine atom nor carboxylic group separation observed.

\section{Conclusions}

The purpose of this study was to separate and identify products created in the photodegradation process of ofloxacin and levofloxacin and to determine the kinetics of the decomposition reactions. In order to achieve that, solutions of ofloxacin and levofloxacin were prepared and irradiated using a mercury-vapor lamp for various time periods up to 210 minutes. Photoproducts separation was carried out using two analytical techniques: a chiral ligand-exchange reversed-phase HPLC and HPCE with 2-hydroxypropylo- $\beta$-cyclodextrin as a chiral selector. It was found that numerous products appeared as a result of photodegradation. Using HPLC method it was possible to observe two products of OFX degradation and only one for LVX, while using an HPCE method eight FPs of OFX and six of LVX were observed. It was concluded that some of the photoproducts still retain character of optically active compounds. It is supposed that during irradiation some amount of (S)-ofloxacin enantiomer is converted into (R)-ofloxacin. The course of photodegradation of the tested compounds is best described by the autocatalytic reaction proceeding according to the Prout-Tompkins model. Some of the products of the decomposition are also catalyzing this reaction. Based on MS experiments it was possible to identify the photodegradation products of the studied fluoroquinolones and possible pathways of UV induced decay.

\section{Acknowledgements}

Conflict of interest statement

The authors declare no conflict of interest.

\section{Funding sources}

There are no sources of funding to declare.

\section{References}

1. Andreu V, Blasco C, Picó Y. Analytical strategies to determine quinolone residues in food and the environment. TrAC Trends Anal Chem. 2007 Jun;26(6):534-556.

2. Reid G, Potter P, Delaney G, Hsieh J, Nicosia S, Hayes $\mathrm{K}$. Ofloxacin for the treatment of urinary tract infec- tions and biofilms in spinal cord injury. Int J Antimicrob Agents. 2000 Feb;13(4):305-307.

3. Cheng FC, Tsai TR, Chen YF, Hung LC, Tsai TH. Pharmacokinetic study of levofloxacin in rat blood and bile by microdialysis and high-performance liquid chromatography. J Chromatogr A. 2002 Jun;961(1):131-136.

4. Hayakawa I, Atarashi S, Yokohama S, Imamura M, Sakano K, Furukawa M. Synthesis and antibacterial activities of optically active ofloxacin. Antimicrob Agents Chemother. 1986 Jan;29(1):163-164.

5. Albini A, Fasani E (editors). Drugs, photochemistry and photostability. Cambridge (UK): Royal Society of Chemistry; 1998.

6. Vasquez Ml, Hapeshi E, Fatta-Kassinos D, Kümmerer $\mathrm{K}$. Biodegradation potential of ofloxacin and its resulting transformation products during photolytic and photocatalytic treatment. Environ Sci Pollut Res Int. 2013 Mar;20(3):1302-1309.

7. Gübitz G, Schmid MG (editors). Chiral Separations: Methods and Protocols. Totowa (NJ, USA): Humana Press;2004.

8. Stalcup AM. Chiral separations. Annu Rev Anal Chem (Palo Alto Calif). 2010;3:341-363.

9. Horstkötter C, Blaschke G. Stereoselective determination of ofloxacin and its metabolites in human urine by capillary electrophoresis using laser-induced fluorescence detection. J Chromatogr B Biomed Sci Appl. 2001 Apr;754(1):169-178.

10. Yamaguchi J, Oguchi $H$, Tokudome $Y$, Katsuyama M. A case of photosensitive drug eruption induced by sparfloxacin. Nishinihon J Dermatol. 1994;56:1146-1149.

11. Yamaguchi J, Oguchi $H$, Tokudome $Y$, Katsuyama M. Three cases of photosensitive drug eruption induced by fleroxacin. Rinsho Hifuka. 1995;49:817-819.

12. Christ W, Lehnert T. Toxicity of the quinolones. In: Siporin C, Heifetz CL, Domagala JM (editors). The New Generation of Quinolones. New York (USA): Marcel Dekker;1990; p. 165-187.

13. Cosa G. Photodegradation and photosensitization in pharmaceutical products: Assessing drug phototoxicity. Pure Appl Chem. 2004;76(2):263-275.

14. Tokura Y. Quinolone photoallergy: photosensitivity dermatitis induced by systemic administration of photohaptenic drugs. J Dermatol Sci. 1998 Sep;18(1):1-10.

15. Budai $M$, Gróf $P$, Zimmer A, Pápai $K$, Klebovich I, Ludányi K. UV light induced photodegradation of liposome encapsulated fluoroquinolones: An MS study. J Photochem Photobiol A. 2008 Aug;198(2-3):268-273.

Acceptance for editing: 2016-12-10 Acceptance for publication: 2016-12-22

Correspondence address:

Prof. Zenon J. Kokot, PhD Department of Inorganic and Analytical Chemistry Poznan University of Medical Sciences 6 Grunwaldzka Street, $60-780$ Poznań, Poland phone: +48 6185466 10, fax: +48 618546609 email: zkokot@ump.edu.pl 